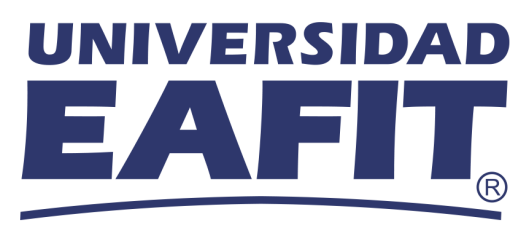

\title{
A pipeline for Solid Domestic Waste classification using Computer Vision
}

Daniel Otero Gómez ${ }^{1}$

\section{Santiago Cartagena Agudeld ${ }^{2}$}

\section{Santiago Isaza Cadavid ${ }^{3}$}

\author{
Advisors: \\ Mauricio Toro Bermúdez 4 \\ Juan Camilo Ramirez De Los Rios 5
}

\author{
Research practice I and II \\ Research proposal \\ Mathematical Engineering \\ Department of Mathematical Sciences \\ School of Sciences \\ Universidad EAFIT
}

August 2021

\footnotetext{
${ }^{1}$ Mathematical Engineering Student at Universidad EAFIT (CvLAC: Daniel Otero Gómez)

${ }^{2}$ Mathematical Engineering Student at Universidad EAFIT (CvLAC: Santiago Cartagena Agudelo)

${ }^{3}$ Mathematical Engineering Student at Universidad EAFIT (CvLAC: Santiago Isaza Cadavid)

${ }^{4}$ IT and Systems Department professor at Universidad EAFIT mtorobe@eafit.edu.co

${ }^{5}$ Mathematical Engineer juan43.ramirez@gmail.com
} 


\begin{abstract}
This work aims to build and analyze a pipeline for solid domestic waste classification. The first steps that were carried out for this were to divide into three main lines that work together to achieve the pipeline. Each line used different sub-approaches to deep learning, relying on both the literature and the advisors, but without neglecting the binary classification work previously carried out. Additionally, a CRISP-DM methodology is taken into account to carry out the work without taking apart the mathematical concepts behind each computationally implemented method, and it is specified what are the intentions of the authors for the near future and their conclusions.
\end{abstract}

Keywords: Image classification, Solid domestic waste, Deep learning, Data augmentation, Transfer learning.

\title{
1 Introduction
}

Solid Domestic Waste (SDW) corresponds to solid elements of day-to-day use that are discarded by households and several commercial and community centers, such as malls, workplaces, universities, etc. SDW is one of the types of post-consumer waste which includes glass, paper, metal, cardboard and trash. The continuous increase in SDW generation is associated with population growth and an increase in consumption which leads to an increase on debris, especially among urban areas. An inter-continental study performed in 22 different countries determined that, in average, households contribute $55-88 \%$ of municipal solid wastes and that market and comercial areas contribute a 10-30\% (Abdel-Shafy \& Mansour, 2018). Since SDW is produced at such a high density and intensity it is crucial that a correct treatment is given to it.

Research indicates that since 19508.3 billion tons of solely plastic were produced. Moreover, 6.3 billion tons were disposed as debris, from which $9 \%$ were recycled, $12 \%$ were incinerated, and $73 \%$ were located in landfills (Geyer et al. 2017). This is highly alarming due to the fact that the expected time of soil destruction of non-organic wastes is quite long. For instance, glass lasts one million years to be completely destroyed, and plastic, depending of the type, may vary from 58 to 1200 years as (Chamas et al. 2020) and (Mohajerani et al., 2017) describe. Appropriate waste management must not be underestimated.

Recycling technologies that are currently employed have limitations on the composition of recyclable waste. In advanced cities, there have been attempts to integrate smart technology into urban areas; the most implemented and discussed idea is a radio-frequency identification (RFID). However, the interaction with this technology must be done locally, which becomes a problem of flexibility and cost and a high environmental impact, see Valente et al. $(2019)$. This enlarges the importance of building an effective and better technological project for the disposal of SDW.

Our main goal is to build a competent pipeline with state-of-the-art techniques that do not require complicated network architectures with hundreds of thousands of parameters. The purpose is that with a smaller amount of data the pipeline has good results when compared to much more complex networks.

Currently, the state-of-art algorithms that dominate the computer vision tasks correspond to big deep learning architectures with large sets of parameters that are trained over millions of images belonging to a broad range of categories. The developments generated from this movements are impressive, however, they are not the only way of achieving high performance models. This work attemps 
The remaining part of the article will be structured as it follows. Section 2 contains a literature review of research papers related to the topic. The review is mainly focused on explaining the GAN background, state-of-art developments in the image classification and object detection domain, and feature extraction techniques used in computer vision problems. The implemented methods and algorithms along the data set are explained in Section 3. In Section 4, a compilation of the experimentation and results can be found. Finally, Section 5 contains the conclusions and future work.

\section{State of the art}

GANs were introduced by Goodfellow et al. (2014) where they proposed a new framework for generative models architecture, with a discriminator model and how to optimize it. The novelty of their development is the adversarial process, in which two models are trained simultaneously, a generative one that captures the distribution of the data and a generative one, which classifies whether a sample comes from the training data's underlying distribution. The procedure when training the generative model consists mainly in maximizing the probability that the discriminator makes an error, so it becomes a min-max game between two players (the models).

These early models worked well but were unstable and difficult to train. Radford et al. (2015) proposed a standardized approach called Deep Convolutional Generative Adversarial Adversarial Networks (DCGAN), which are much more stable. Many of the GANs today have an infrastructure similar to the one proposed. The infrastructure is, in general, determined by two sub-models: a model to generate new examples and a discriminator model to classify whether the generated examples are real (from our domain or dataset) or fake (product of the generator model).

Examples of the use of Deep Learning for data synthesis are several, for example, a team from the Deep Learning class at Stanford University used a fast R-CNN to detect objects and solid debris by comparing a baseline model trained on the trashnet dataset using GAN-generated images to augment the dataset used Kulkarni \& Sundara Raman (2019). Although the main objective of the team was the detection of objects and their subsequent classification the use of artificially generated images improved performance, in particular, precision, recall and f1-score. Unfortunately the authors do not specify how these results were corroborated whether it was in training or validation.

This methodology of data augmentation is being widely used, for example, Frid-Adar et al. (2018) generated synthetic medical images using GANs. They proposed a training scheme where they first used traditional data augmentation techniques to enlarge the training set and then they applied GAN techniques to enlarge the data size and its diversity. The results were astonishingly good. The classification performance with classic techniques yielded $78.6 \%$ sensitivity and $88.4 \%$ specificity. When the synthetic data was added the results increased to $85.7 \%$ sensitivity and $92.4 \%$ specificity.

Currently, machine learning algorithms are one of the main tools for solving classification tasks and the idea of employing this tool for waste sorting is not new. Generally speaking, there are two key points in such works: a model, that is tested, and a database for training and testing of the network. Given that state-of-the-art methods for detecting objects of general classes are mainly based on deep CNNs, a noteworthy effort has been carried out in the past years in the detection of objects using convolutional neural networks (CNNs), Girshick et al. (2014).

In the object detection and recognition field, neural networks are in use for a decade but became prominent due to the improvement of hardware new techniques for training these networks on large 
datasets, see Ren et al. (2015). Earlier publications reported testing of classical convolutional neural networks, Yang \& Thung (2016). The CNNs in the field of computer vision, regarding object detection, can be split into two types: region-proposal-based and regression/classification-based. Regression/classification-based CNNs have better-performing networks with respect to computational cost while maintaining a level of accuracy that is on par with other CNNs.

A more recent advancement in neural networks design is the so-called region proposal convolutional neural network (R-CNN). This model incorporates different blocks for the detection of objects in images and the classification of the detected objects. In this case, multiple objects of different types can be recognized in one image. There have been few examples of successful employment of region-proposal neural networks for waste sorting. One of such works is Zhihong et al. (2018), in this work authors tested an R-CNN-type neural network which was trained on a dataset of 1332 scenes with multiple labeled objects in the scene. The authors reported classification precision of $78.5 \%$, however, the paper contains very few details about the dataset, training, and testing procedures.

Girshick et al. (2014) proposed a multi-stage pipeline, denoted as regions with convolutional neural networks (R-CNN), for training deep CNNs in order to classify region proposals for object detection. It decomposes the detection problem into several stages, including bounding-box proposal, CNN pre-training, CNN fine-tuning, SVM training, and bounding box regression.

A better algorithm that tackles the issue of predicting accurate bounding boxes while using CNNs is the YOLO algorithm, see Sai et al. (2019). YOLO stands for you only look once which was developed in 2015 by Joseph Redmon, Santosh Divvala, Ross Girshick, and Ali Farhadi. It is popular because it achieves high accuracy while running in real-time. The last-mentioned algorithm is the one that is going to be implemented throughout this work.

\section{Methodology}

\subsection{The dataset}

The data set was extracted from a public repository that can be found in here. It contains 2390 images of six different classes: cardboard, glass, metal, paper, and plastic. The image size is $384 \times 512$ and they are stored in .jpg format. The cardboard class contains 403 images, paper contains 594 images, metal contains 410 images, plastic contains 482 images, glass contains 501. Achieving a good classification performance given the amount of images available to train the model seems as a big challenge, however, techniques such as data augmentation pipelines, stacking routines, and transfer learning generated feature vector are implemented here in order to enhance the models' learning and assess the improvements made in comparisson with previous experiments. A sample of the images is shown below. 


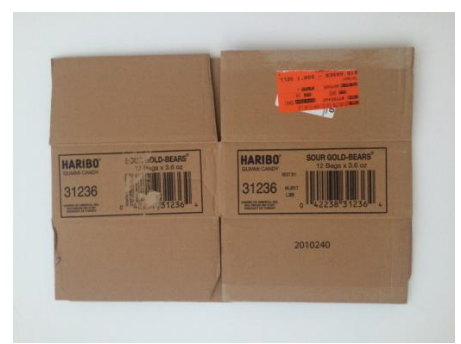

Figure 1: Cardboard image sample.

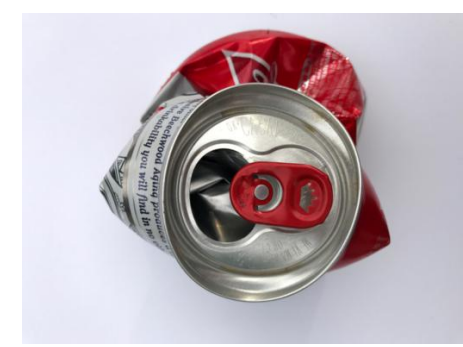

Figure 2: Metal image sample.

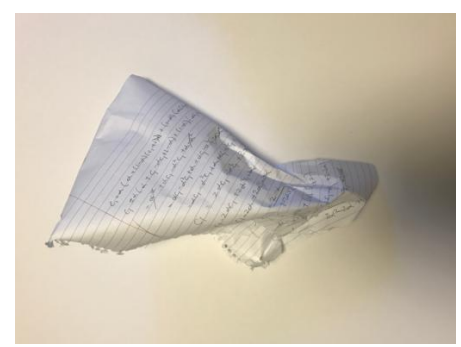

Figure 3: $\quad$ Paper image sample.

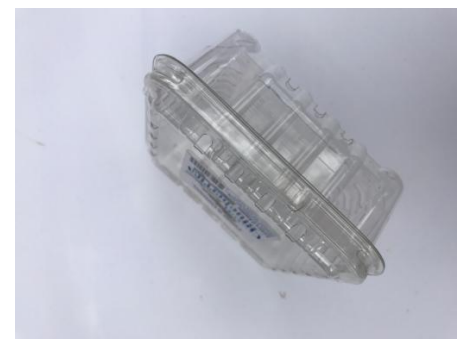

Figure 4: Plastic image sample.

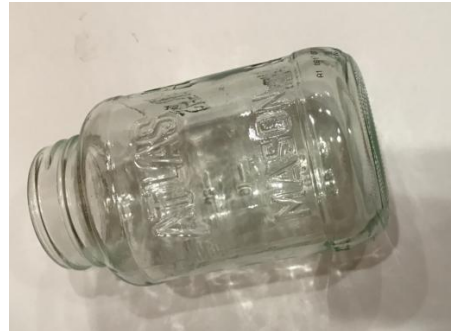

Figure 5: Glass image sample.

\subsection{Generative Adversarial Network}

For Data Augmentation the main purpose was to use Generative Adversarial Networks and classic techniques like cropping and rotating images in order to augment the current dataset, this to better regularize the better classifier already trained by Otero et al. (2021). To develop the GAN it was used tensorflow and keras with python, and the GAN approach was changed to use a AC-GAN better to generate multiclass images like solid domestic waste is (glass, metal, cardboard, etc.). The architecture of our AC-GAN can be seen in figure 6. The main idea of an AC-GAN is to connect a neural netowrk classifier to the generator network in order to help it generate images of a specific class, for example metal. In our modelling first we changed the real/fake hard labels in our discriminator pipeline to soft labels. We also added noise by randomly flippling the labels, this to help boost the generator, as described in Bhattarai et al. (2020). The GAN literature recommended using LeakyLeLU for both the generator and discriminator models. In the experiments this approach indeed showed improvements. 


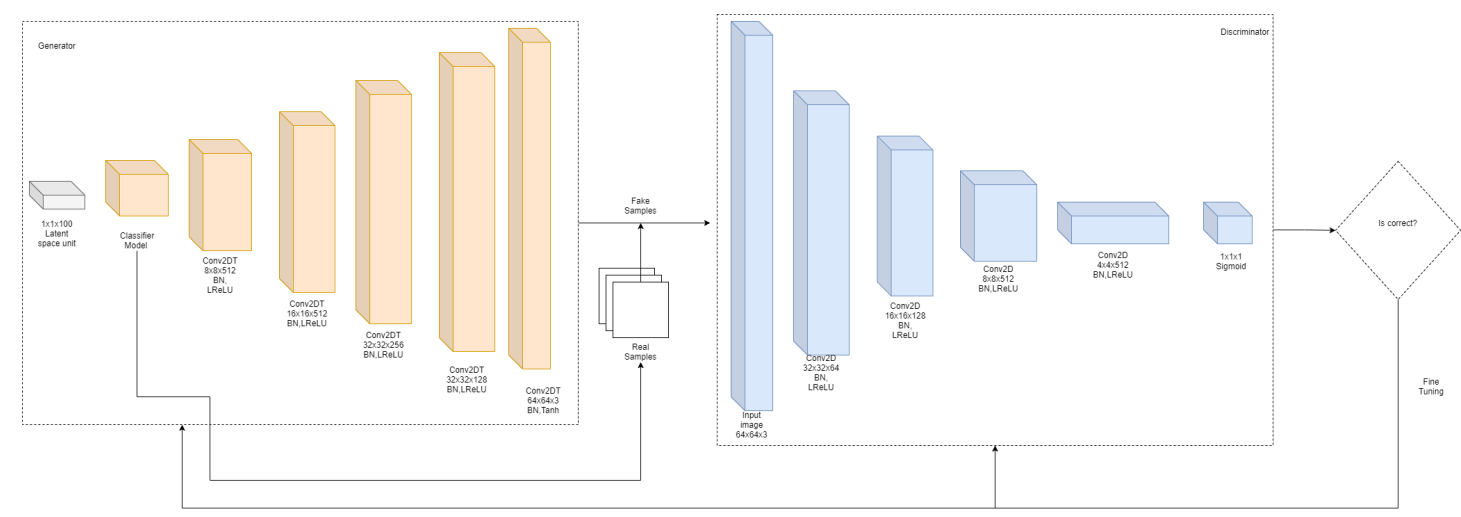

Figure 6: AC-GAN architecture

The dataset used for training our network is the TACO dataset, see Proença \& Simões (2020). We used a subset composed of 2557 images divided in six classes: glass, paper, cardboard, plastic, metal and trash. For each of them we used 501,594, 482, 410 and 137 images respectively. The realized experiments consisted in three experimentation with two hyperparameters: the number of layers, the number of channels for layer. Our baseline generator network has a decreasing number of channels in each layer, this is: $512 \rightarrow 256 \rightarrow 128 \rightarrow 64 \rightarrow 3$. Additionally, changing the architecture to use a large number of channels in the initial layers: $512 \rightarrow 512 \rightarrow 512 \rightarrow 128 \rightarrow 3$, this helped the network learn more feautres and showed improvements in the generator output.

Next, we tried increasing the number of layers by introducing $3 x 3$ deconvolution filters in the generator network. After this we tried multiple permutations and combinations, for example, introducing $3 \times 3$ layer at the end. Based on the experiments and the results a significative improvement was noticed after adding the described layer in the final part of the network compared to other combinations. The results matched our intuition, a deconvolutional layer at the start of the generator network does not help because the initial layers is still learning features and outputs noise domninant imagws, while adding deconvolutional layers at the end provide better results because at higher layers the network is still learning features of the dataset. Once data for every class was generated, over 150 new images for everey class, we used it to train and evaluate the performance of Otero et al. (2021) best previous model, a support vector machine classifier. We realized three evaluations: with the original dataset, with an augmented dataset with classic techniques (cropping and rotating) and finally with the GAN augmented dataset.

\subsection{Image Classification Experimentation Process}

Firstly, the performance of the pipeline developed in Otero et al. (2021) was contrasted with Deep Learning approaches to the problem. Otero et al. (2021) take into account a bounded version of the task in which carboard and metal images were considered solely. Hence, a CNN and a Transfer Learning model were trained and the results were compared. The simpler version of Google's EfficientNet was used to draw that upper limit to which the developments made among the research is heading.

Afterward, a full version of the problem was considered and classification pipelines were developed to face the multi-class task. It is important to note that the results achieved in the two different versions of the problem are not comparable since the complexity of the five-class classification task 
is much higher than the binary problem. Otherwise, important improvements to the model may be looked past.

A baseline was set first by using the same pipeline developed in Otero et al. (2021) and used to classify the five classes. Then, stacking was considered as a new classification procedure and several meta learners were tested to find the best performing meta learner and increase the performance. After this, data augmentation techniques were introduced to the baseline models and the stacking procedures in order to accurately assess that it has a significant impact on the evaluation metrics. Finally, the EfficientNet B0 was used in three different ways to compare the achieved results: the feature vector constructed by the last layer before the output of the model was passed to the models in order to check if the relations characterized by EfficientNet were useful for the models to learn, the top layer of the model was replaced for it to classify the considered labels, and a five-layer fine-tuning was performed.

Finally, the Macro F1 score was selected because of its higher sensibility to misrepresented classes in comparisson with the accuracy score. For instance, if one of the five classes is completly misclassified but the other four are perfectly classified the accuracy will be affected in much less proportion than the Macro F1 score. This metric sensibility will allow to get a better grasp of how well is the classifier performing without looking at the confusion matrix.

\subsection{Object Detection Tasks}

Aiming to perform object detection tasks, it was decided to use a popular algorithm known as YOLO which is an algorithm that uses neural networks to provide real-time object detection. This algorithm is popular because of its speed and accuracy, since it uses a predictive technique that provides accurate results with a minimum of background errors, $\mathrm{Du}(2018)$. Besides, the algorithm is able to detect and recognize various objects in a picture (in real-time) using Convolutional Neural Networks. Object detection in YOLO is done as a regression problem and provides the class probabilities of the detected images. The way in which this algorithm works is through the use of three main techniques, which are residual blocks, bounding box regression and Intersection Over Union (IOU).

First, for the residual blocks part, the image is divided into several grids. Each grid has a dimension of $\mathrm{S} \times \mathrm{S}$. The following image shows how an input image is divided into grids. 


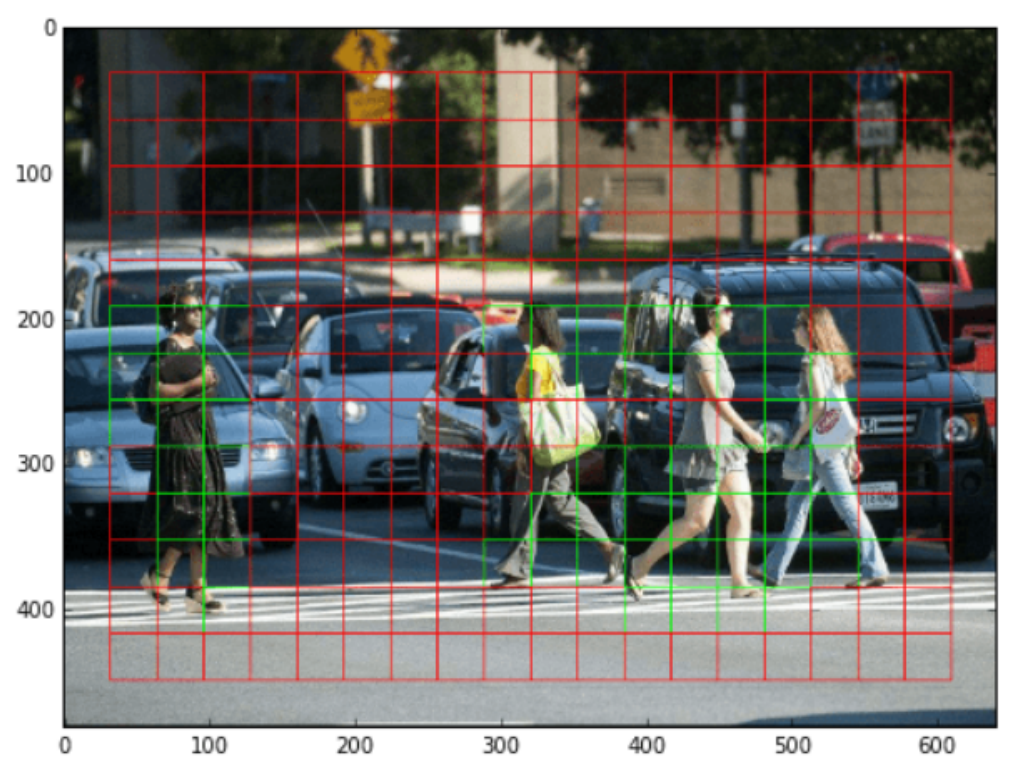

Figure 7: Input image divided into grids

In the image above, there are many grid cells of equal dimension. Every grid cell will detect objects that appear within them. For example, if an object center appears within a certain grid cell, then this cell will be responsible for detecting it.

Second, a bounding box is an outline that highlights an object in an image. Each bounding box in the image consists of the following attributes: width, height, and class. YOLO uses a single bounding box regression to predict the height, width, center, and class of objects.

Finally, Intersection over Union (IOU) is a phenomenon in object detection that describes how frames overlap. YOLO uses IOU to provide an exit box that surrounds objects perfectly. Each cell in the grid is responsible for predicting the bounding boxes and their confidence scores. The promissory note equals 1 if the expected bounding box is the same as the actual box. This mechanism removes bounding boxes that are not the same as the actual box.

\section{Results}

\subsection{Data Augmentation Experimentation}

In figure 8 is easy to observe that the proposed $3 x 3$ deconvolution kernel helps the layer map learned features, resulting in a better generator output. Aditionally to generate the data our model trained for over 30 hours. However, as the image shows there are some repeated images in the generated grid. We suspect that this is just a random sampling issue however it is possible that the GAN model collapses. This should be further investigated and can be an interesting future work. The results of the loss function for the training process can be seen in figure 9 . 

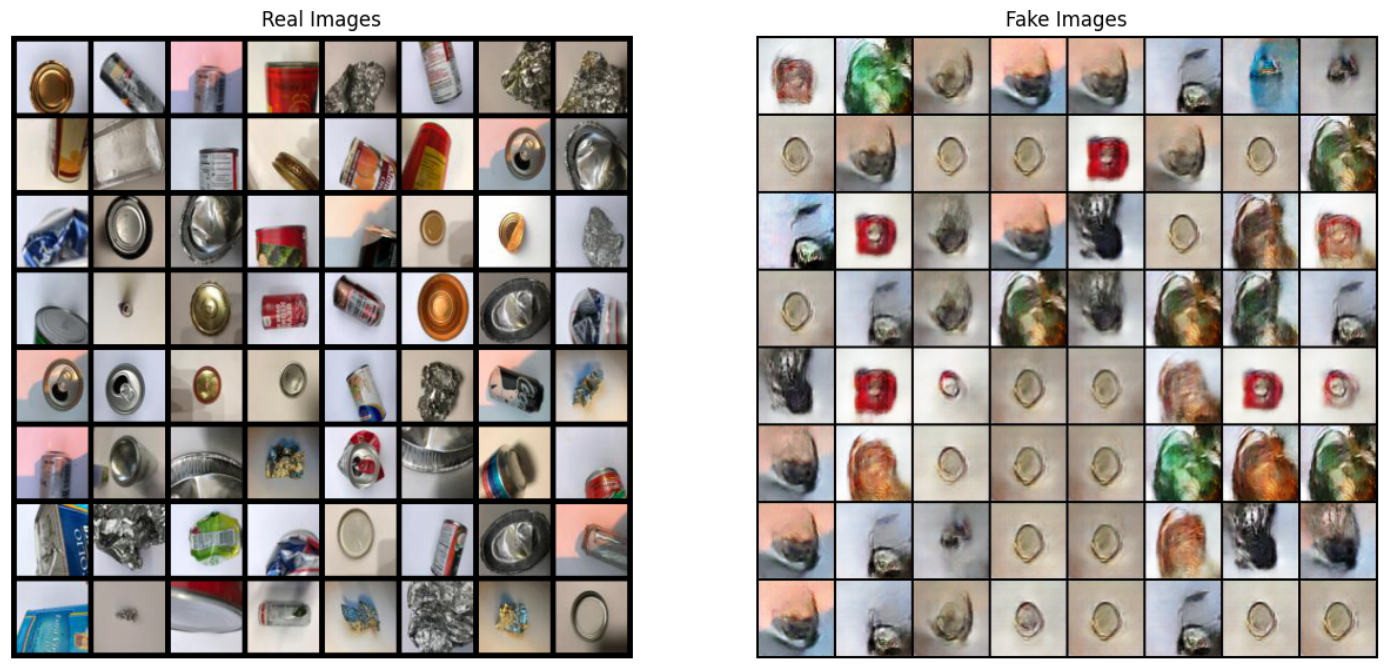

Figure 8: Generated images

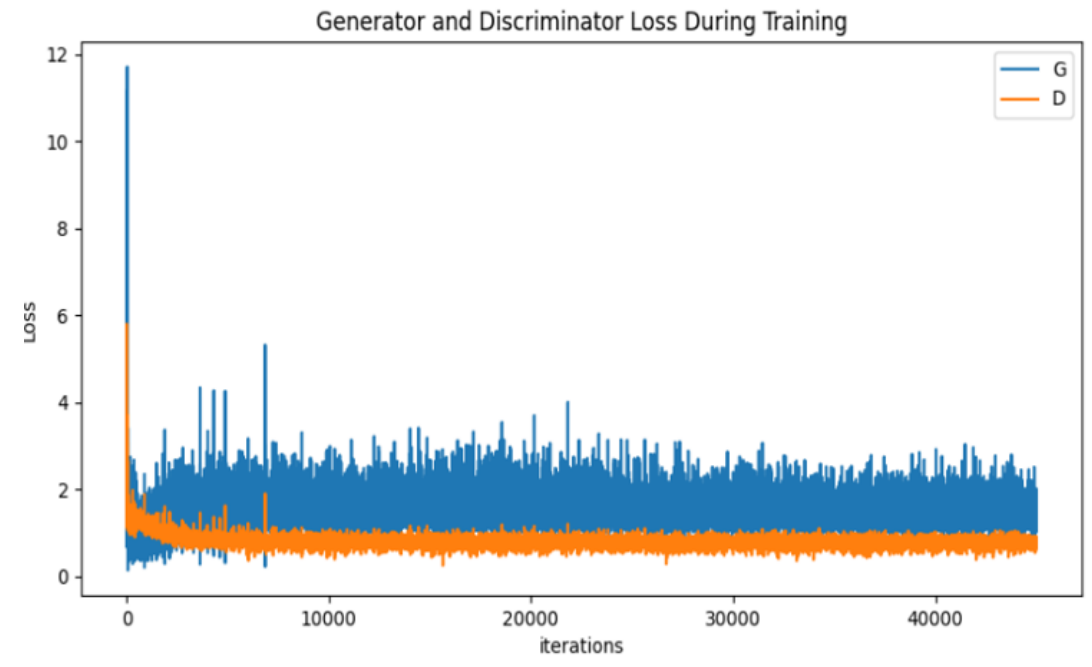

Figure 9: Loss function of the generator and discriminator models

Figure 10 is the result of a $4 x 4$ kernel and a reduced dataset experimentation. This in order to see the performance of the model with a large neighborhood and a reduced dataset. Opposed to the results in figure 8, with this experimentation almost every generated images looks fake. Other conducted experiment was using filters in the beginning of the network, switching our $4 x 4$ filter to a $2 x 2$ deconvolutional kernel resulted in a drop of the performance. As we can see below in figure 11 the fake images were extremely noisy, with not a distinguishable feature. The plot 12 shows this as the generator has high loss values. 

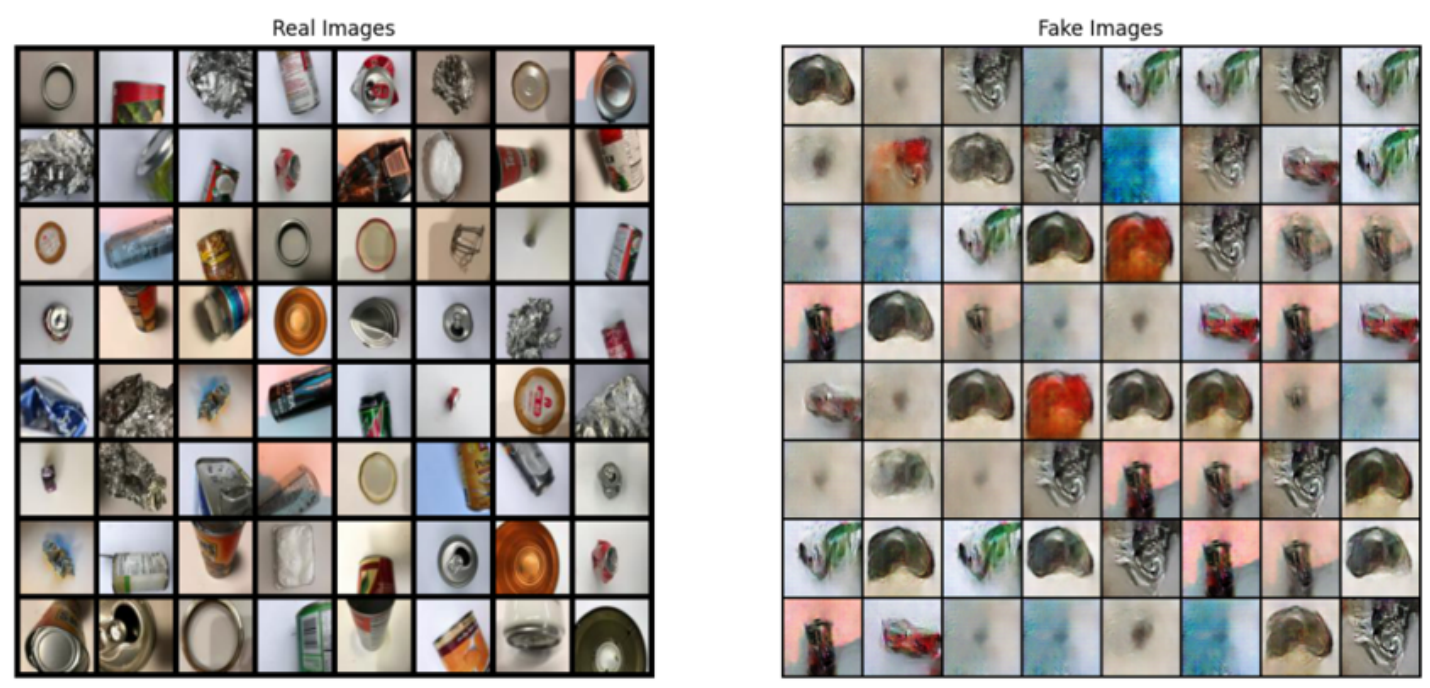

Figure 10: Reduced dataset generated images
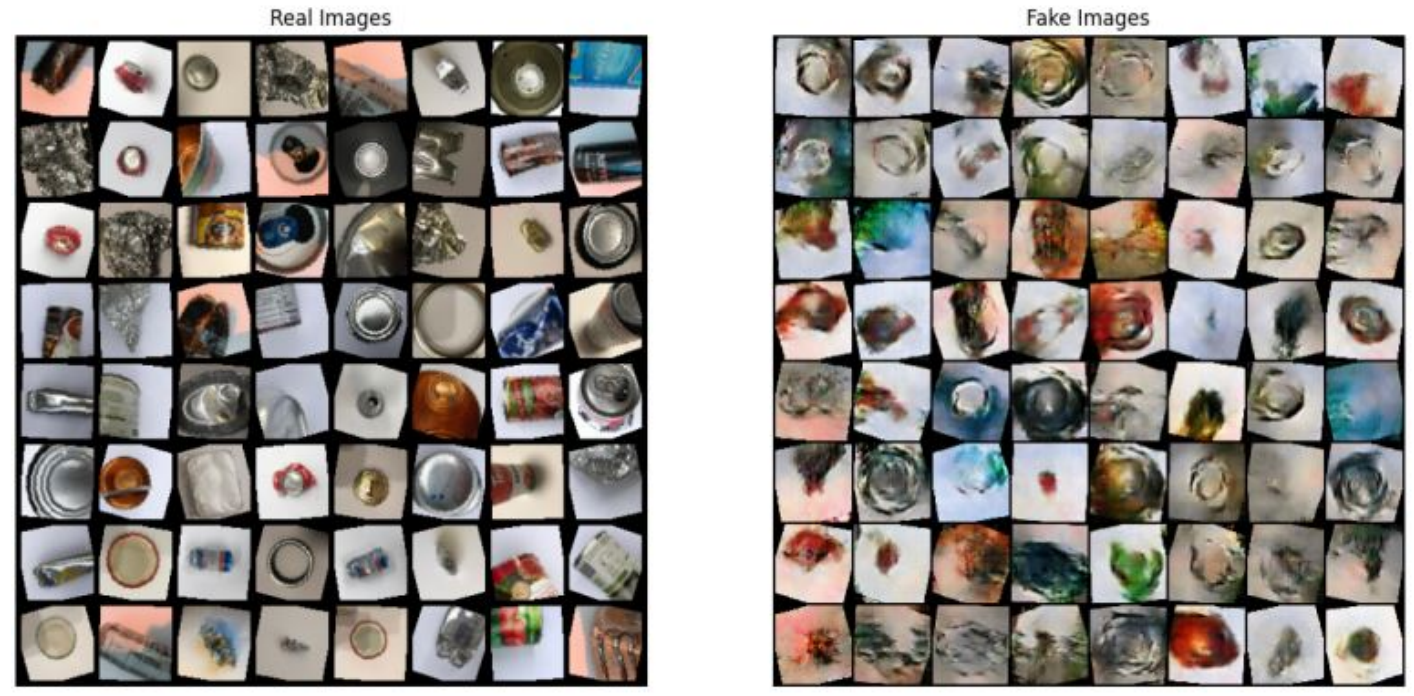

Figure 11: Generated images with a $2 x 2$ filter 


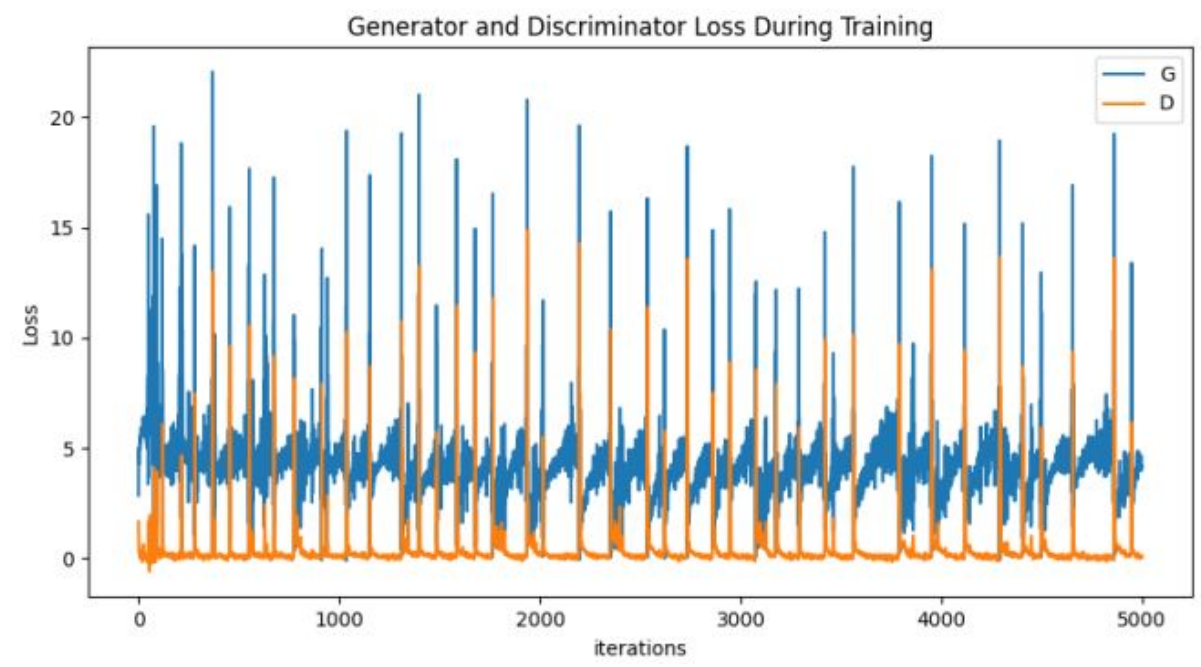

Figure 12: Loss function of the generator and discriminator with a $2 x 2$ filter

The majority of the generated dataset doesn't have the expected quality. This is clearly seen when the dataset is used to train and validate Otero's classifier Otero et al. (2021). In figure 13 different metrics are compared between datasets, specifically accuracy and area under a ROC curve. Evidently the best results were obtained by the classic augmented dataset, while our artificially augmented one didn't perform well. Thus, it is correct to conclude that the majority of the GAN generated images doesn't have the quality desired. However, some results are really promising, showing the potential of a well trained and optimized Generative adversarial network. As a ROC curve analysis was also held, figure 14 shows the respective curves for each dataset.

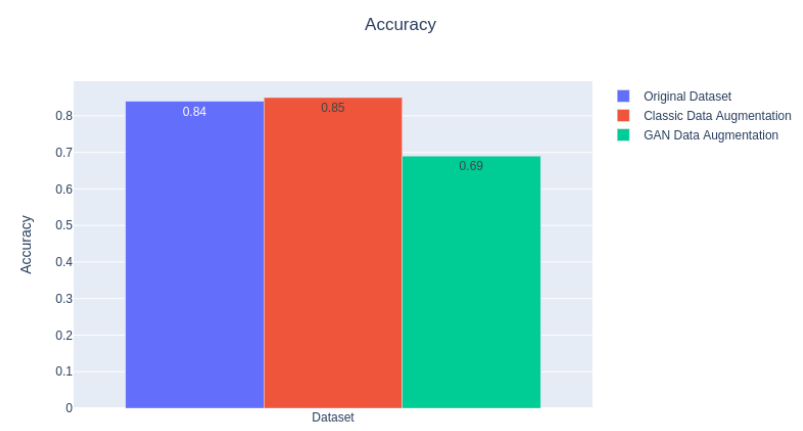

(a) Accuracy

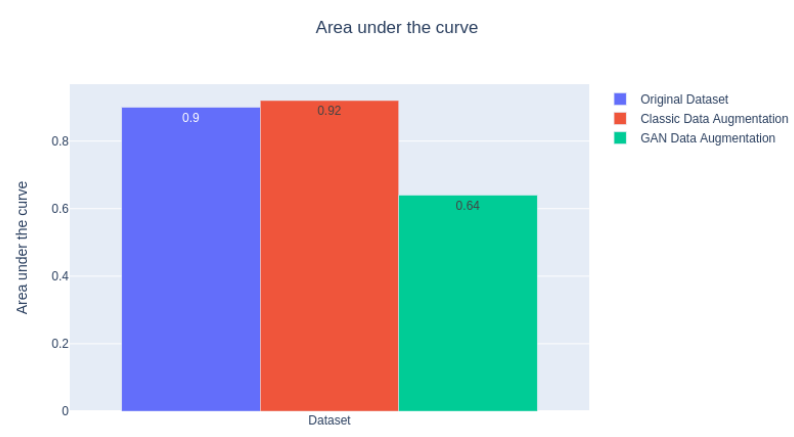

(b) Area under curve

Figure 13: Metrics for the classifier with different dataset

An analysis of the ROC curve was also performed, see figure 14, and its respective area in figure 13. It is evident that the model trained with traditional data augmentation techniques is the best. It is also clear that the images generated by the GAN did not have the desired behavior, as we see how a portion of the curve is under the threshold. It is quite possible that this happens due to biases in the distribution of the learned data. For example, generating circular elements when the 
expected object is from the class of metallic objects, a characteristic that although this class also shares with plastic bottles or paper cups.

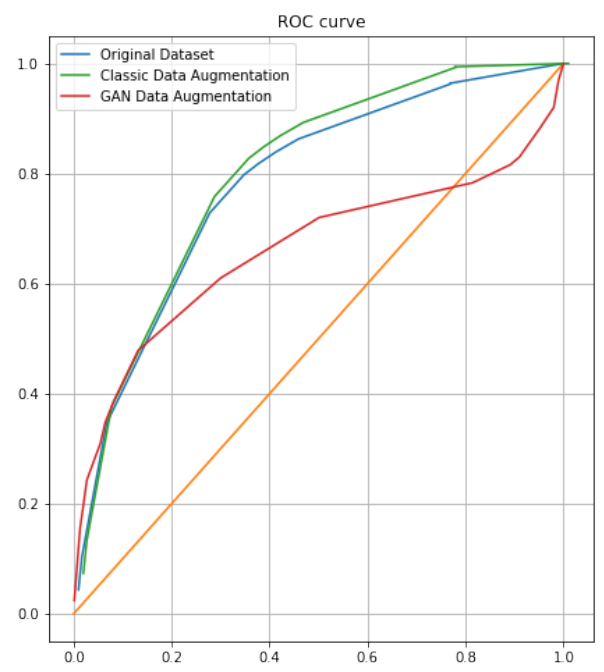

Figure 14: ROC Curve for each dataset

\subsection{Image Classification Experimentation}

In Table 1 the baseline results may be found. The "Learning" column refers to the fit that the learning curves show throughout the experimentation. It may be observed that every model tested shows a good fit, except for the Random Forest, which shows to have been underfitting the data. This indicates that the generalization ability of the model is poor, and even though it shows good results it is not guaranteed that the performance is consistent across datasets. Moreover, high complexity algorithms have superior performance than simpler algorithms such as the Logistic Regression or the Naive Bayes. In addition, the AdaBoost and Support Vector Machine learning curves indicate that the models may keep improving in the presence of more data, things that may be satisfied by applying data augmentation. This is exhibited in Figure 15 as the curves are monotone and do not fully stationed nor touch. Now, the lack of complexity that the Logistic Regression and the Naive Bayes show may be fixed by applying stacking procedures to build a higher complexity learner composed of several sub-models.

\begin{tabular}{||c|c|c||}
\hline Model & Macro F1 & Learning \\
\hline Ada Boost - Decision Tree - depth=8 & 0.5995 & Good Fit \\
\hline Random Forest & 0.5975 & Bad Fit \\
\hline Support Vector Machine & 0.5761 & Good Fit \\
\hline Logistic Regression & 0.5161 & Good Fit \\
\hline Gaussian Naive Bayes & 0.5161 & Good Fit \\
\hline
\end{tabular}

Table 1: Single Model Approach - Baseline 


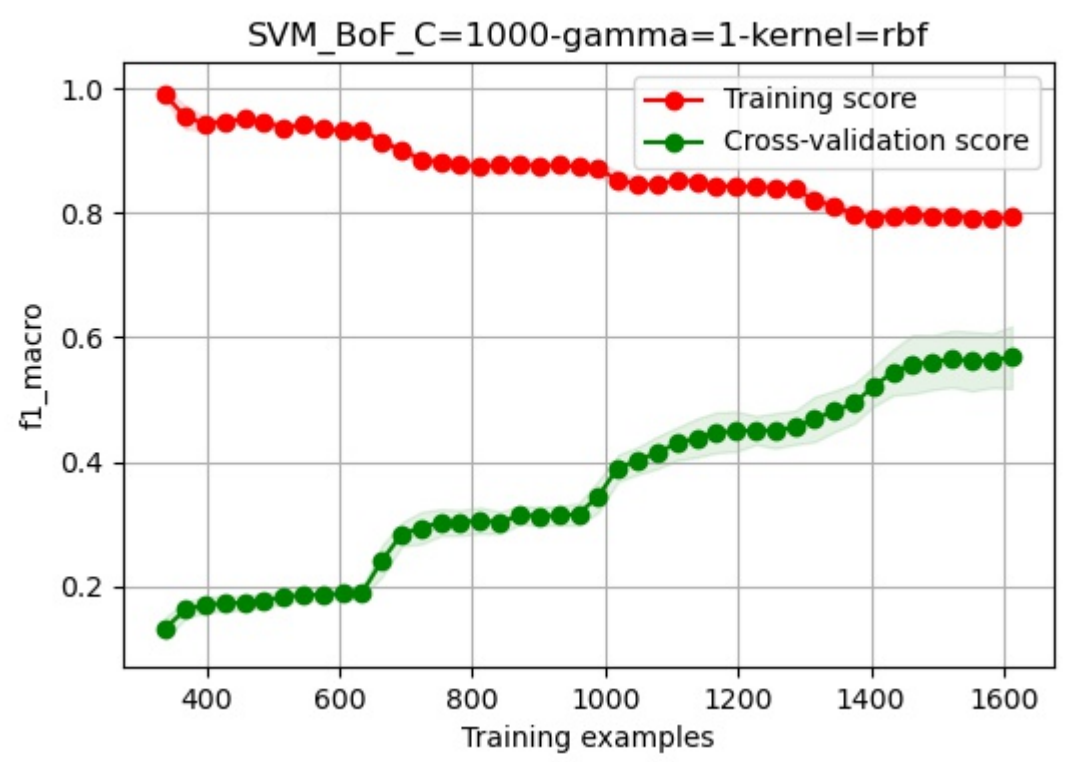

Figure 15: Support Vector Machine Baseline learning curve.

The stacking procedure was implemented in the way that every tested model predicted the probability of an image the belong to all the classes. Then these probabilities were appended to the built feature vector for the meta-learner to use them as significant features. In this way, a new dataset was construed using the Random Forest, Logistic Regression, Support Vector Machine, Gaussian Naive Bayes, and AdaBoost as sub-models and the performance was once again tested using each of these models as the meta learners. In Table 2 the stacking results may be observed.

\begin{tabular}{||c|c|c||}
\hline Model & Macro F1 & Learning \\
\hline Random Forest & 0.6253 & Good Fit \\
\hline Logistic Regression & 0.6188 & Good Fit \\
\hline Support Vector Machine & 0.6148 & Good Fit \\
\hline Single Layer Perceptron - activation=softmax & 0.6103 & Good Fit \\
\hline Ada Boost - Decision Tree - depth=8 & 0.6109 & Bad Fit \\
\hline Gaussian Naive Bayes & 0.5831 & Good Fit \\
\hline
\end{tabular}

Table 2: Stacking Method Performance

Now, the results show that the Random Forest learning was enhanced by the procedure. The Macro F1 score increased by $3 \%$ approximately. More importantly, the complexity increment of the classification method appears to have a positive impact on the Logistic Regression and Naive Bayes performances. The Macro F1 score increased by $10 \%$ and $7 \%$ respectively. This is a very positive result given the complexity of the problem, the limited dataset, and the simplicity and ease of use and interpretation of the models. On the other hand, higher complexity models are not affected very significantly since the metric improvement is the same as shown by the Random Forest. Furthermore, the bad fit exhibited implies that boosting technique used within the AdaBoost technique is affected by the combination of different ensemble techniques. This may not be the case 
for the Random Forest since stacking is a similar procedure to Bagging, and choosing the Random Forest as a meta-learner simulates a deeper "forest" structure.

\begin{tabular}{||c|c|c||}
\hline Model & Macro F1 & Learning \\
\hline Random Forest & 0.6093 & Bad Fit \\
\hline Ada Boost - Decision Tree - depth=8 & 0.5985 & Bad Fit \\
\hline Support Vector Machine & 0.5598 & Good Fit \\
\hline Logistic Regression & 0.4982 & Good Fit \\
\hline Gaussian Naive Bayes & 0.4650 & Good Fit \\
\hline
\end{tabular}

Table 3: Data Augmentation - Single Model Approach

Now, considering the experimentation detailed in the previous subsection, classical data augmentation techniques were applied to enlarge the dataset and verify if this has a positive impact on the model performance. As it may be seen in Table 3 the data augmentation technique does not have a positive initial impact over the single-model approach. It provoked a decrease in the none tree-based estimators and it ended underfitting the AdaBoost classifier, which was not underfitting previously. It is easy to suspect that this is caused due to bad implementation techniques, however, taking a look at Table 4 we can appreciate indeed that data augmentation has a positive impact on the performance of the model. The reason for which the single models perform poorly is that the image transformations have been done in a way in which simpler models cannot understand the new data relations. A more sophisticated, however, more difficult, approach such as GAN-based data augmentation may solve this problem.

\begin{tabular}{||c|c|c||}
\hline Model & Macro F1 & Learning \\
\hline Random Forest & 0.6405 & Bad Fit \\
\hline Logistic Regression & 0.6387 & Good Fit \\
\hline Support Vector Machine & 0.6369 & Good Fit \\
\hline Ada Boost - Decision Tree - depth=8 & 0.6341 & Bad Fit \\
\hline Gaussian Naive Bayes & 0.5921 & Good Fit \\
\hline
\end{tabular}

Table 4: Data Augmentation - Stacking Method Performance

\begin{tabular}{||c|c|c||}
\hline Model & Macro F1 & Learning \\
\hline Support Vector Machine & 0.8442 & Bad Fit \\
\hline Logistic Regression & 0.8201 & Bad Fit \\
\hline Random Forest & 0.7886 & Bad Fit \\
\hline Gaussian Naive Bayes & 0.7096 & Good Fit \\
\hline
\end{tabular}

Table 5: Transfer Learning Feature Vector

Moreover, observing the results obtained by using the feature vector generated by the EfficientNet B0 at the penultimate layer. We can see that most of the classifiers are not complex enough to understand the relations that the feature vector resembles. This is happening due to its large dimensions (more than 2,000). Due to the ability to work with the high-dimensional-large-data of 
the Gaussian Naive Bayes, the model is capable of achieving the highest metric yet. This serves as a more realistic upper limit that these techniques may offer us within the conditions considered since it still takes advantage of the Transfer Learning power without using large algorithms, millions of parameters, and enormous training datasets.

To further compare the methods developed with the transfer learning of the EfficientNet B0 a feature vector and fine-tuning version of them were trained. Since the fine-tuning results are related to the feature vector version of the model, for simplicity reasons, the joint loss curves will be shown but only the fine-tuning confusion matrix of the fine-tuning model will be exhibited.

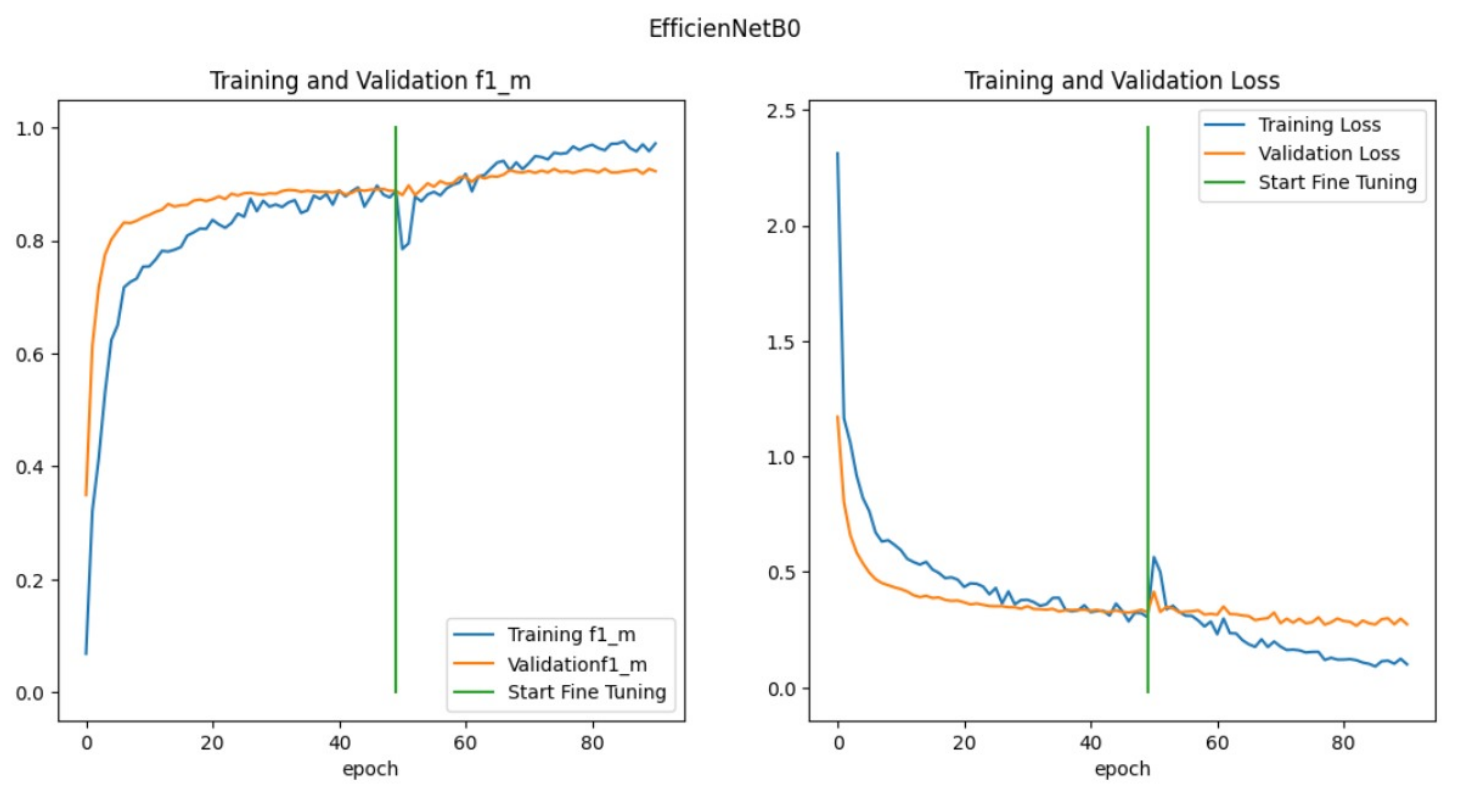

Figure 16: Feature Vector and Fine-Tuning loss curves.

During the feature extraction phase, the model shows an atypical behavior where the validation loss and metrics are better than the training ones. This happens because the layers remain static and the knowledge gained from the images is only being stored in the output layer. During the fine-tuning phase, this behavior stops and it acquires a normal behavior. Moreover, in Figure 17 the confusion matrix has a great performance with a significantly low percentage of mislabeled images. In Figure 18 and 19, the confusion matrix of the best classifier yet developed along the feature vector trained Naive Bayes is appended as well to illustrate better the final comparison. 

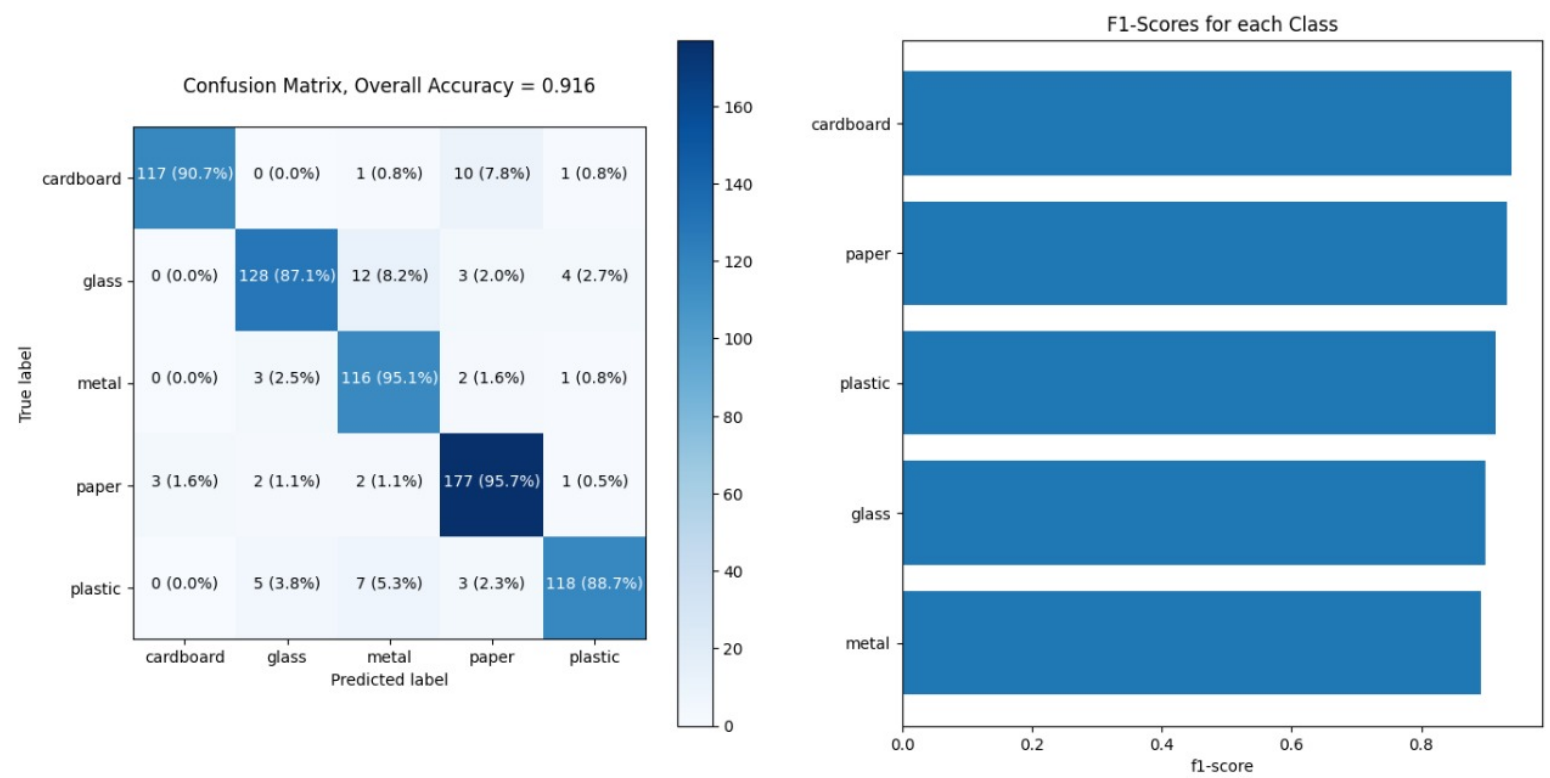

Figure 17: Confusion Matrix and F1 Barplot of fine-tuning model.

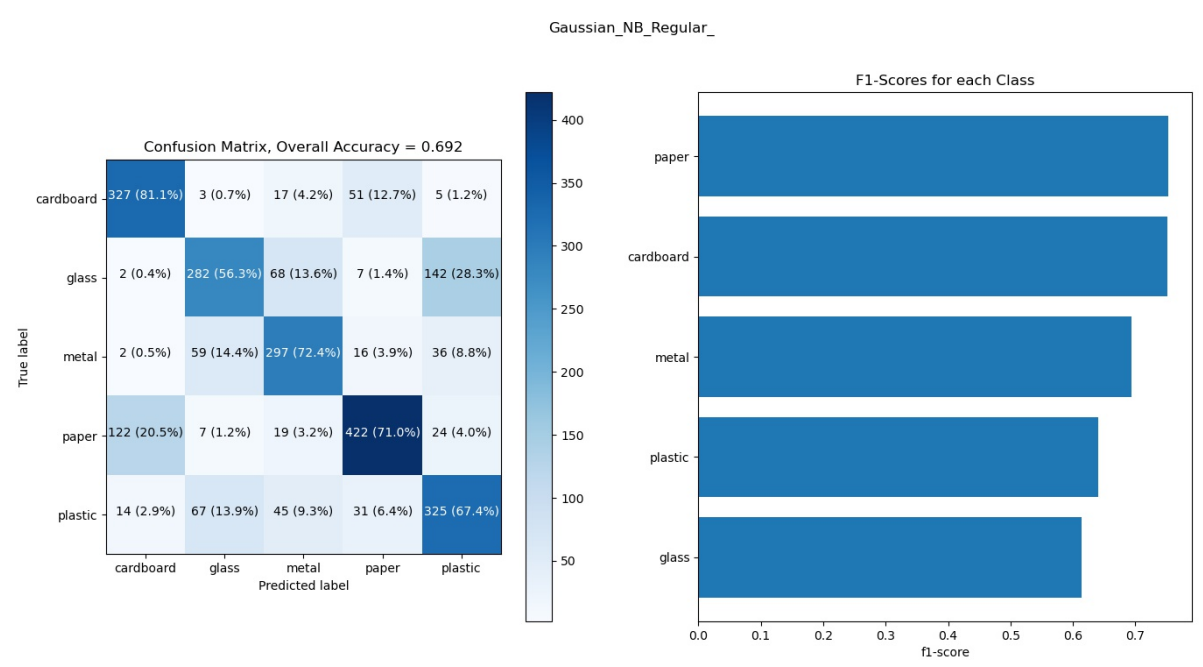

Figure 18: Confusion Matrix and F1 Barplot of feature vector trained Naive Bayes. 


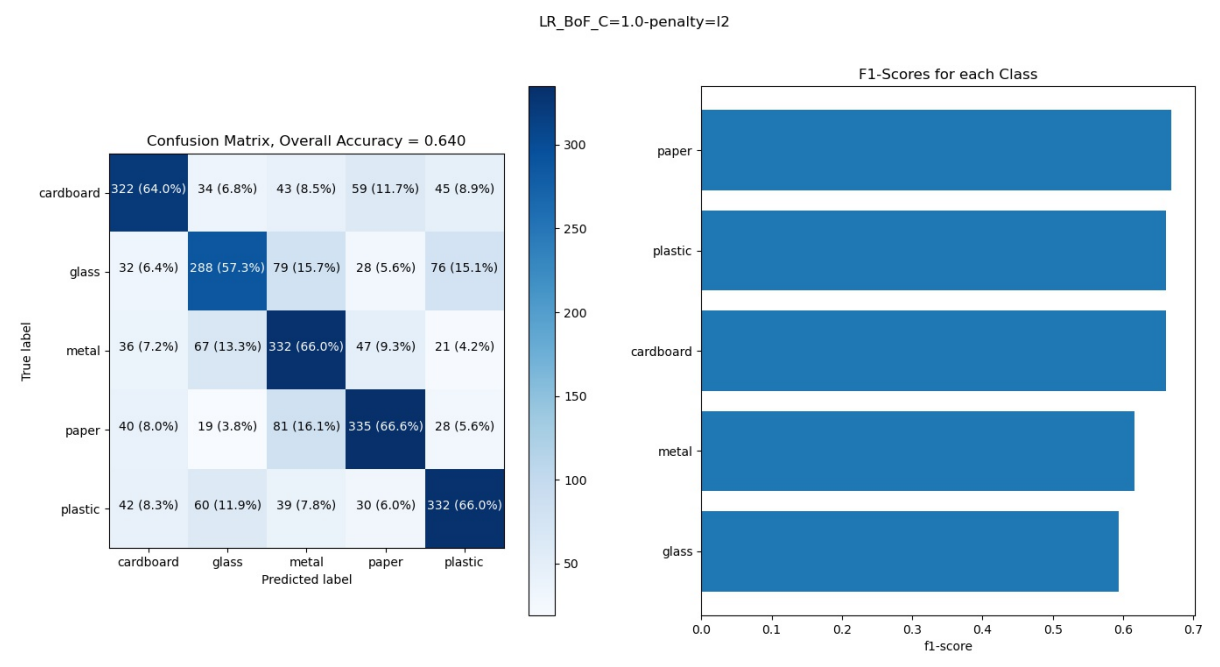

Figure 19: Confusion Matrix and F1 Barplot of data augmentation stacking Logistic Regression.

\subsection{Object Detection Experimentation}

In figure 18 you can see part of the aforementioned methodology that is used by the YOLO object detection algorithm, this image was obtained thanks to a mixture between YOLO and COCO, which is a data set named after its acronym Common Objects in Context, this dataset contains 80 classes including car, person, motorcycle, dog, cat, carrot, etc.

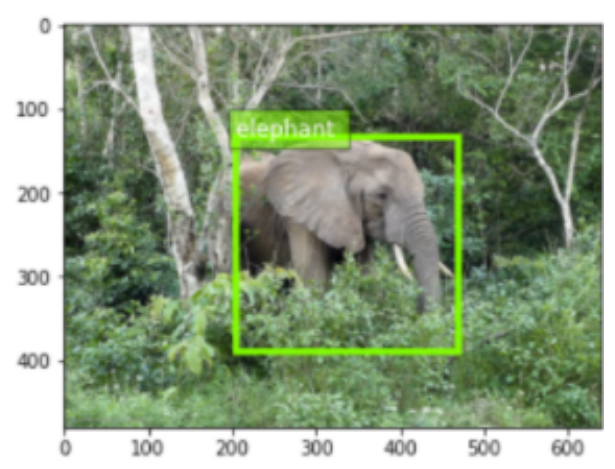

Figure 20: First Object Detection Approach.

After this, we now enter the matter strongly with the part of the detection of objects with the aforementioned combination YOLO + COCO, as can be seen in figure 19, in which we have not only the location of the objects in the image but also the probability of belonging of these objects to the classes available in the COCO data set. 


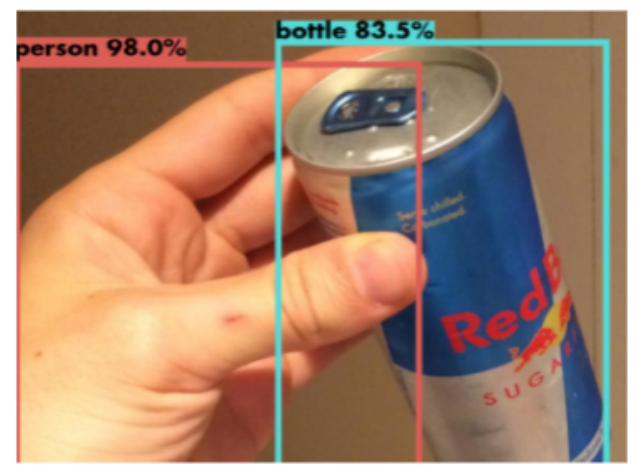

Figure 21: Second Object Detection Approach.

Continuing with this detection of objects, we now have a task applied to SDW images, this in order to bring the neural network built from the YOLO algorithm closer and closer to our problem of multiclass classification of household solid waste and thus be able to start making performance comparisons with Otero's classifier.

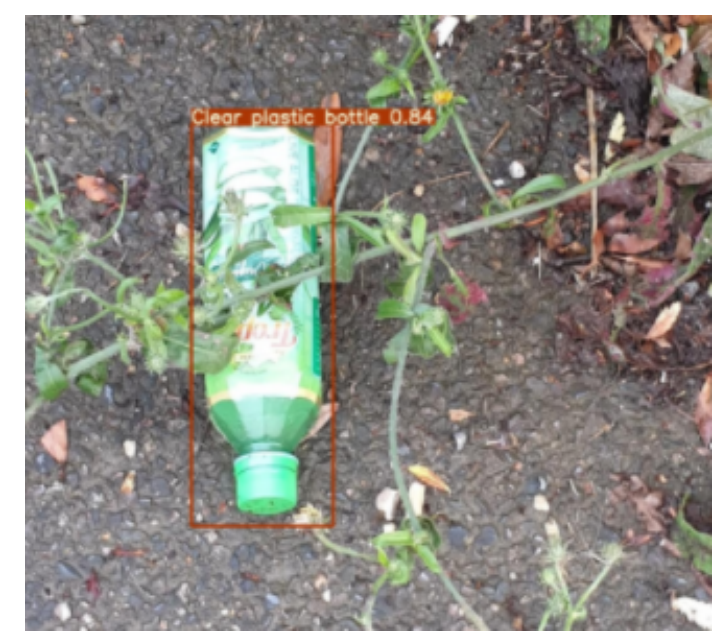

Figure 22: Third Object Detection Approach (Closer to our classification problem).

In order to see the performance in terms of accuracy of the YOLO neural network, a plot was created to observe the loss function and accuracy in detail, both were quite good considering that we are working with a multiclass classification, which makes prediction a bit more difficult. It is important to note that given the precision and speed mentioned by YOLO, it is largely possible to obtain such good results and with high confidence when making predictions. 

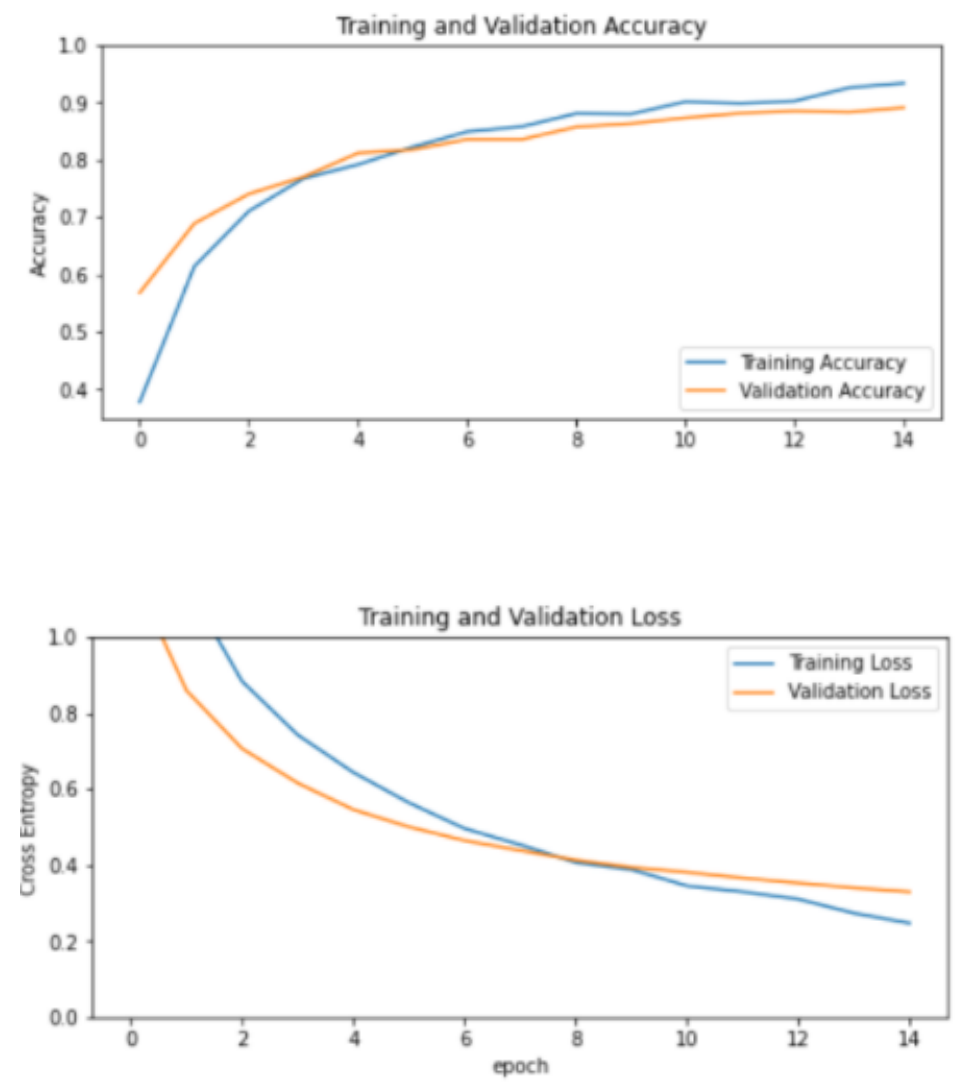

\section{Conclusions and future research}

In this research, we tried to use a state-of-the-art technique for data augmentation, generative adversarial networks. Based on our preliminary results GAN generated images can have biased distributions that can cause collapses in the model as seen in the results section and better described in Shu et al. (2017). Additionally, we see several key approaches for future work. First, use state of the art pre-trained models to help reduce runtime overall (as our training phase took more than 30 hours and generating data takes a significant amount of time) also use transfer learning techniques to improve our generative models as well as cloud technologies like Amazon Web Services or Google Cloud. Moreover, we would like to use explore several architeCtures as the one described in this work was built with an empirical approach. Another important consideration is the fact that classic data augmentation techniques outperformed the gan generated dataset, thus these techniques should never be discarded.

Regarding the image, classification experimentation was performed. It has been shown that the stacking procedure in combination with data augmentation techniques is a very powerful method in order to improve machine learning models' performance. The idea behind stacking that allows to create high complexity, good performing models, from low complexity low performing models is paired perfectly with the possibility of generating larger datasets from small ones. In order to further improve the performance of the models the BoF approach can be perfected by implementing state-of-art methods such as the SURF and SIFT methodologies. Furthermore, a larger data 
augmentation with more complex generated images may lead to an improvement with simple models, and consequently, have a larger impact on the stacking models or other abstraction implementation that is chosen.

In terms of Object Detection in conjunction with image classification and transfer learning techniques, a great performance was obtained as expected in terms of precision, thanks in large part to the chosen algorithm and hyperparameter tuning, all this still being a multiclass problem, which in the first instance intuitively it was expected that it would not give such satisfactory results.

\section{Acknowledgements}

Special thanks to our advisor Mauricio Toro Bermúdez and Juan Camilo Ramirez De los Rios who guided us through all this process and answered to all of our questions.

\section{References}

Abdel-Shafy, Hussein, \& Mansour, Mona. 2018. Solid waste issue: Sources, composition, disposal, recycling, and valorization. Egyptian Journal of Petroleum, 27(12), 1275-1290.

Bhattarai, Binod, Baek, Seungryul, Bodur, Rumeysa, \& Kim, Tae-Kyun. 2020. Sampling strategies for GAN synthetic data. Pages 2303-2307 of: ICASSP 2020-2020 IEEE International Conference on Acoustics, Speech and Signal Processing (ICASSP). IEEE.

Chamas, Ali, Moon, Hyunjin, Zheng, Jiajia, Qiu, Yang, Tabassum, Tarnuma, Jang, Jun Hee, Abu-Omar, Mahdi, Scott, Susannah, \& Suh, Sangwon. 2020. Degradation Rates of Plastics in the Environment. ACS Sustainable Chemistry Engineering, 1(02).

Du, Juan. 2018. Understanding of object detection based on CNN family and YOLO. 1004(1), 012029 .

Frid-Adar, Maayan, Klang, Eyal, Amitai, Michal, Goldberger, Jacob, \& Greenspan, Hayit. 2018. Synthetic data augmentation using GAN for improved liver lesion classification. Pages 289-293 of: 2018 IEEE 15th international symposium on biomedical imaging (ISBI 2018). IEEE.

Geyer, Roland, Jambeck, Jenna, \& Law, Kara. 2017. Production, use, and fate of all plastics ever made. Science Advances, 3(07), e1700782.

Girshick, Ross, Donahue, Jeff, Darrell, Trevor, \& Malik, Jitendra. 2014. Rich feature hierarchies for accurate object detection and semantic segmentation. Pages 580-587 of: Proceedings of the IEEE conference on computer vision and pattern recognition.

Goodfellow, Ian, Pouget-Abadie, Jean, Mirza, Mehdi, Xu, Bing, Warde-Farley, David, Ozair, Sherjil, Courville, Aaron, \& Bengio, Yoshua. 2014. Generative adversarial nets. Advances in neural information processing systems, $\mathbf{2 7}$.

Kulkarni, N. Hrushikesh, \& Sundara Raman, Nandini Kannamangalam. 2019. Waste Object Detection and Classification. http://cs230.stanford.edu/projects_fall_2019/reports/ 26262187.pdf. 
Mohajerani, Abbas, Vajna, John, Cheung, Tsz, Kurmus, Halenur, \& Arulrajah, Arul. 2017. Practical recycling applications of crushed waste glass in construction materials: A review. Construction and Building Materials, 156(12), 443-467.

Otero, D., Toro, M., \& Hoyos, W. 2021. Solid Domestic-Waste (SDW) Classification using Image Processing and Machine Learning.

Proença, Pedro F, \& Simões, Pedro. 2020. TACO: Trash annotations in context for litter detection. arXiv preprint arXiv:2003.06975.

Radford, Alec, Metz, Luke, \& Chintala, Soumith. 2015. Unsupervised representation learning with deep convolutional generative adversarial networks. arXiv preprint arXiv:1511.06434.

Ren, Shaoqing, He, Kaiming, Girshick, Ross, \& Sun, Jian. 2015. Faster r-cnn: Towards real-time object detection with region proposal networks. Advances in neural information processing systems, 28, 91-99.

Sai, Mukkamala R., Miguel, \& Veeravelli, Sainagesh. 2019. OBJECT DETECTION AND IDENTIFICATION A Project Report. ResearchGate.

Shu, Rui, Bui, Hung, \& Ermon, Stefano. 2017. Ac-gan learns a biased distribution. In: NIPS Workshop on Bayesian Deep Learning, vol. 8.

Valente, Miguel, Silva, Hélio, Caldeira, Joao M., \& Gaspar, Pedro D. 2019. Detection of waste containers. Instituto de Telecomunicacoes, Universidad de Beira Interior.

Yang, Mindy, \& Thung, Gary. 2016. Classification of trash for recyclability status. CS229 Project Report, 2016.

Zhihong, Chen, Hebin, Zou, Yan, Wang, Yanbo, Wang, \& Binyan, Liang. 2018. Multi-task Detection System for Garbage Sorting Base on High-order Fusion of Convolutional Feature Hierarchical Representation. 5426-5430. 